\title{
The different tiling of 12-fold rosettes in Moroccan geometric art
}

Youssef Aboufadil $^{1}$, Abdelmalek Thalal ${ }^{2}$, Khaoula Ait Rai ${ }^{2}$, Abdelaziz Jali ${ }^{2}$, My Ahmed El idrissi Raghni ${ }^{2}$, Amane Oueriagli ${ }^{2}$ ${ }^{1}$ Department Of Physics, Polydisciplinary Faculty Safi, Cadi Ayyad University, Marrakech, Morocco, ${ }^{2}$ Department of Physics, Faculty of Sciences Semlalia, Cadi Ayyad University, MARRAKECH, Morocco

E-mail: y.aboufadil@gmail.com

Moroccan decorative art, especially geometric art or "Tastir" is one of the most complex and one of the most refined expressions of Arab-Islamic art. The rosettes may be the essential elements of the ornamental panel or be used as a repetitive element to construct an infinite periodic tiling.

Periodic tilings of the plane are Moorish ornamental tradition (Andalusia and the Maghreb), which culminated between the 13 th and 14 th centuries.

This presentation is devoted to the study of 12 -fold rosettes frequently found in Islamic art in general and Moroccan in particular. There are several 12-fold rosettes types according to the geometric shape of its petals. They have a large number of variants and also offer the possibility of building new rosettes previously unknown, while respecting the construction method "Hasba" used by master craftsmen (Thalal \& Al, 2011; Aboufadil \& Al, 2013).

The craftsmen have developed a technique for combining different types of 12 -fold rosettes to construct infinite periodic tilings belonging to the 17 crystallographic groups. This technique enabled them to diversify the repeated patterns based on 12-fold rosettes. By analysing their tilings, we propose a method based on elementary geometry and symmetry to build new patterns with different types of rosettes and their variants. We also present a procedure, based in combination of the distances between two overlapped rosettes, which simplifies the method of construction used by artisans and which enables to generate new periodic patterns as well as and quasi-periodic.

Aboufadil, Y. \& Thalal, A. \& Raghni, M. A. E. I. (2013). Symmetry groups of Moroccan geometric woodwork patterns, J. Appl. Cryst. 46, 1834-1841.

Aboufadil, Y. \& Thalal, A. \& Elidrissi Raghni, M. A. (2014). Moroccan ornamental quasiperiodic patterns constructed by the multigrid method, J. Appl. Cryst. 47, 630-641.

Thalal, A. \& Benatia, M. J. \& Jali, A. \& Aboufadil, Y. \& Elidrissi Raghni, M. A. (2011). Islamic geometric patterns constructed by craftsmen working on wood. Symmetry: Culture and Science Vol. 22, Nos. 1-2, 103-130.

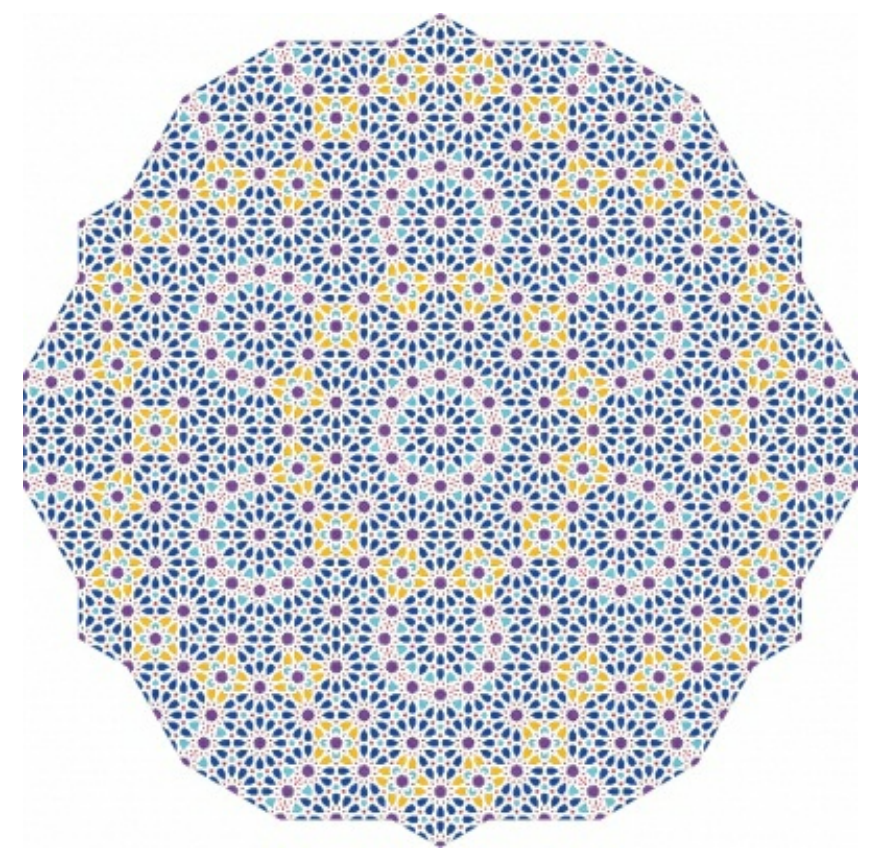

Keywords: 12 -fold rosettes, symmetry, geometric pattern 